\title{
Role of calmodulin in blastocyst formation in the mouse
}

\author{
P. L. Pakrasi and S. K. Dey \\ University of Kansas Medical Center, Ralph L. Smith Research Center, Department of Obstetrics \\ and Gynecology and Physiology, Kansas City, Kansas 66103, U.S.A.
}

\begin{abstract}
Summary. Preimplantation mouse embryos were recovered by flushing the oviducts on Day 3 at $09: 30-10: 00 \mathrm{~h}, 15: 30-16: 00 \mathrm{~h}$ and $21: 30-22: 00 \mathrm{~h}$ : When placed in culture for $48 \mathrm{~h}, 79 \%$ of the $4-8$ cell embryos recovered at $09: 30-10: 00 \mathrm{~h}$ developed into blastocysts, but a large number of these embryos failed to form blastocysts when exposed to trifluoperazine, a calmodulin antagonist, at 0.5 or $0.6 \mu \mathrm{M}$ in culture. About $45 \%$ of the embryos recovered at 15:30-16:00 h were compacted and blastocyst formation was again markedly depressed in the presence of the drug. Advanced compacted embryos recovered at 21:30-22:00 h showed normal development into blastocysts in the presence of $0.6 \mu \mathrm{M}$-trifluoperazine. Trifluoperazine sulphoxide (the inactive form of trifluoperazine) at 0.6 or $1.2 \mu \mathrm{M}$ concentration had no effect on blastocyst formation of uncompacted embryos recovered at $09: 30-10: 00 \mathrm{~h}$. These embryos and those recovered at $21: 30-22: 00 \mathrm{~h}$ and developed into blastocysts in the presence of trifluoperazine were transferred to Day-4 pseudopregnant mice and healthy young were born. When exposed to calcium-free medium or medium containing trifluoperazine all compacted embryos recovered at $18: 30 \mathrm{~h}$ became decompacted; development to the blastocyst stage was normal in medium alone but reduced when trifluoperazine was added. Compacted embryos recovered at $23: 00 \mathrm{~h}$ showed $100 \%$ decompaction in the calcium-free medium but completely failed to decompact in the presence of $0.6 \mu \mathrm{M}$-trifluoperazine. We suggest that extracellular calcium is essential for the continuance of compaction, while intracellular calcium is required only during the initial phase of this process.
\end{abstract}

\section{Introduction}

The process of compaction is prerequisite for morula to blastocyst transformation. In the mouse the process begins at the 8-cell stage and is associated with remarkable morphological changes (Ducibella \& Anderson, 1975). However, the exact mechanism(s) involved in the process is not clearly understood. Of the several possibilities (Surani, 1979; Pratt, Keight \& Chakraborty, 1980; Atienza-Samols, Pine \& Sherman, 1980; Hudgins, Mukherjee \& Dey, 1982), the role of extracellular calcium in compaction is well established. On the other hand, the information regarding the influence of intracellular calcium in this process is limited. The importance of calmodulin-dependent intracellular calcium in the compaction process in the mouse embryo has been indicated (Bilozur \& Powers, 1982), but the role of intracellular calcium at different stages of compaction was not evaluated. In the present investigation, we have studied the role of extracellular and intracellular calcium at different stages of compaction and subsequent blastocyst formation. 


\section{Materials and Methods}

Outbred female mice of Charles River strain (CD-1) were mated by males of the same strain. They were maintained in temperature- $\left(25 \pm 2^{\circ} \mathrm{C}\right)$ and light- $(14 \mathrm{~h}$ light : $10 \mathrm{~h}$ dark) controlled quarters with free access to laboratory food and tap water. The morning of finding the vaginal plug was designated as Day 1 of pregnancy. Mice were killed in the morning (09:30-10:00 h), afternoon $(15: 30-16: 00 \mathrm{~h})$ and evening $(21: 30-22: 00 \mathrm{~h})$ of Day 3 of pregnancy. Embryos were recovered by flushing the oviducts with Whitten's medium (Whitten, 1971) under sterile conditions. The embryos were pooled from several animals, washed 4 times in the same medium and divided into several batches ( $5-16$ embryos $/ \mathrm{ml} /$ tube) for culture. The embryos recovered in the morning were cultured for $48 \mathrm{~h}$, while those recovered in the afternoon or evening were cultured for $41 \mathrm{~h}$ or $36 \mathrm{~h}$. The methods of culture and examination of embryonic development were as reported by Dey \& Johnson (1980). The first set of experiments was designed to determine the effects of trifluoperazine, an antagonist to calmodulin (Connor, Levin \& Brownstein, 1982; Prozialeck \& Weiss, 1982) on blastocyst formation. As shown in Text-fig. 1, the embryos were cultured in the presence of (1) trifluoperazine (Smith Kline \& French Laboratory, Philadelphia, PA) and (2) trifluoperazine sulphoxide (Smith Kline \& French), the inactive form of trifluoperazine (Weiss \& Wallace, 1980). Groups of embryos cultured in the medium alone were always run with the treated embryos to serve as controls. To determine embryo viability and normality, the embryos which developed to blastocysts in the presence and absence of trifluoperazine or trifluoperazine sulphoxide were transferred (3-12 blastocysts/mouse) to Day-4 pseudopregnant mice produced by sterile matings with vasectomized males. The young were observed at birth and until after weaning.

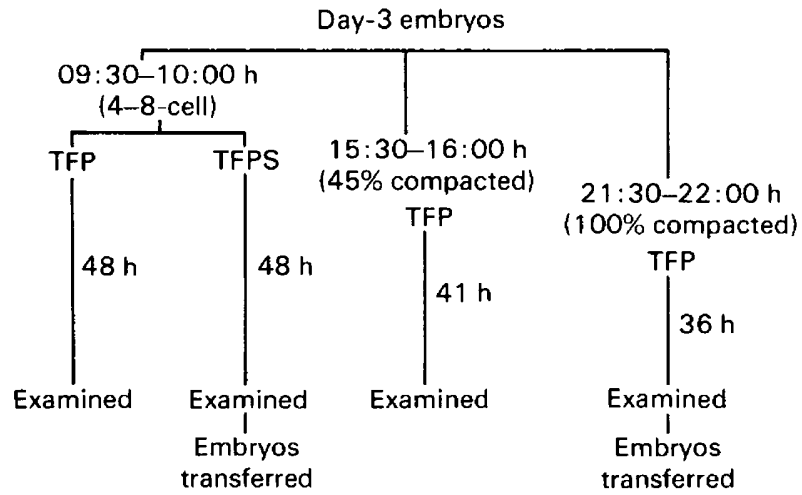

Text-fig. 1. Experimental design to determine the effects of trifluoperazine (TFP) and trifluoperazine sulphoxide (TFPS) on the in-vitro development of mouse embryos recovered at different times on Day 3 of pregnancy. The embryos recovered at $09: 30-10: 00 \mathrm{~h}, 15: 30-16: 00$ $\mathrm{h}$ and $21: 30-22: 00 \mathrm{~h}$ were cultured for $48 \mathrm{~h}, 41 \mathrm{~h}$ and $36 \mathrm{~h}$ respectively. At the end of culture, the embryos were examined under a microscope to count the number of blastocysts formed. In two cases, as indicated, blastocysts developed in the presence of TFP or TFPS were subsequently transferred to synchronized pseudopregnant mice to determine the viability of the blastocysts. Groups of embryos cultured in the medium alone were always run with the treated embryos to serve as controls. Blastocysts developed under normal conditions were also transferred to synchronized recipients.

The second set of experiments was designed to determine the effects of trifluoperazine on embryo compaction. Embryos were recovered from Day-3 pregnant mice at 18:30 h and 23:00 h. Most of the embryos (60\%) recovered at 18:30 h and all of the embryos at 23:00 h were compacted. As shown in Text-fig. 3 compacted embryos were then exposed to calcium-free medium or medium containing trifluoperazine for $2 \mathrm{~h}$ to induce decompaction in vitro. The decompacted embryos thus 
obtained from each treatment were then cultured separately for another $40 \mathrm{~h}$ in the presence and absence of trifluoperazine to determine embryo development under these conditions. The compacted embryos recovered at 23:00 h were exposed to the calcium-free medium or the medium containing trifluoperazine only for $2 \mathrm{~h}$ to study the decompaction.

The results were compared by $\chi^{2}$ analysis.

\section{Results}

As shown in Text-fig. 2, about $80-100 \%$ of the Day-3 embryos recovered at any time developed to blastocysts under our culture conditions. However, addition of trifluoperazine at a concentration of 0.5 or $0.6 \mu \mathrm{M}$ drastically reduced the development of the Day-3 embryos recovered at $9: 30-10: 00 \mathrm{~h}$, while trifluoperazine sulphoxide, even at $1 \cdot 2 \mu \mathrm{M}$ concentration, did not affect embryo development. The development to the blastocyst stage of the Day-3 embryos recovered at 15:30-16:00 $\mathrm{h}$ was also reduced to about $49 \%$ and $22 \%$ by trifluoperazine at concentrations of 0.5 and $0.6 \mu \mathrm{M}$, respectively. This $49 \%$ development to the blastocyst stage could have resulted from the $45 \%$ of embryos already compacted at this time. The development of the embryos recovered at $21: 30-22: 00 \mathrm{~h}$ was not affected by trifluoperazine.



Text-fig. 2. Percentage of blastocysts developed from embryos recovered at different times on Day 3 of pregnancy under various experimental conditions. Values are mean \pm s.e.m.; the numbers inside the bars indicate the number of embryos cultured and numbers in parentheses represent the number of experiments. ${ }^{*} P<0.05$ compared with control values.

From the 53 embryos that developed to blastocysts in medium only and were transferred to 7 foster mothers, 35 healthy offspring were born $(9 / 12,9 / 11,1 / 4,3 / 3,5 / 7,2 / 5,6 / 11)$. A similar transfer of 34 blastocysts, developed from Day-3 embryos recovered at $21: 30-22: 00 \mathrm{~h}$ in the presence of $0.6 \mu \mathrm{M}$-trifluoperazine, to 5 pseudopregnant mice produced 19 healthy young $(4 / 6,3 / 7$, $5 / 5,2 / 8,5 / 8)$. Furthermore, blastocysts which developed from Day-3 embryos recovered at $9: 30$ $10: 00 \mathrm{~h}$ in the presence of 0.6 or $1.2 \mu \mathrm{M}$-trifluoperazine sulphoxide produced healthy offspring when transferred to foster mothers $(3 / 4,6 / 10,7 / 10$ and $7 / 12,6 / 12,7 / 12)$.

The compacted embryos recovered on Day 3 at $18: 30 \mathrm{~h}$ resulted in $100 \%$ decompaction when exposed to calcium-free medium or medium containing trifluoperazine (Text-fig. 3). These decompacted embryos when cultured in the medium alone showed normal development to the blastocyst stage, while addition of trifluoperazine in the medium drastically reduced embryo development. The compacted embryos recovered at 23:00 h, although showing 100\% decompaction in the calcium-free medium, completely failed to decompact in the presence of trifluoperazine (Text-fig. 3). 


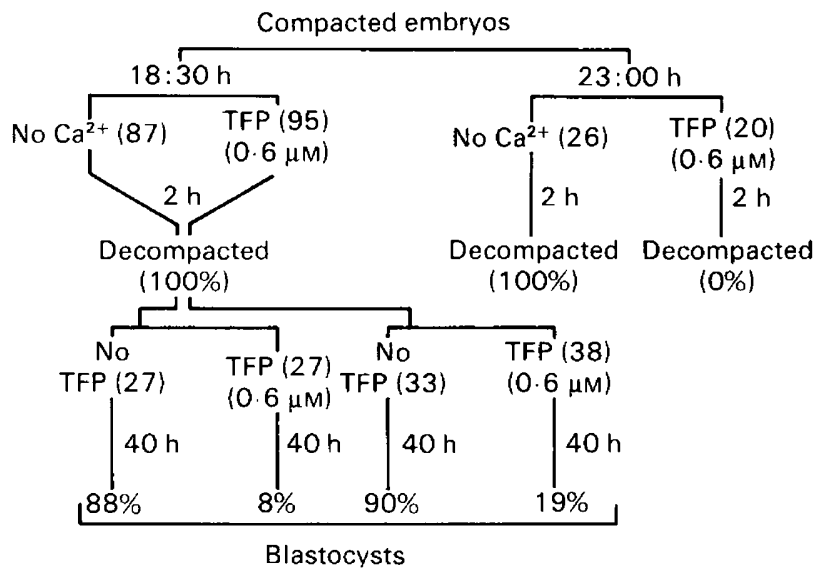

Text-fig. 3. Experimental protocol to determine decompaction and subsequent development of the decompacted embryos after exposure to medium containing TFP or calcium-free medium. Numbers in parentheses indicate the number of embryos for each experiment. Experiments were repeated 5 times except one experiment with TFP at 23:00 h which was repeated twice. The compacted embryos recovered at $18: 30 \mathrm{~h}$ were exposed to the calcium-free medium or the medium containing TFP for $2 \mathrm{~h}$ for inducing decompaction. The decompacted embryos derived from each treatment were then cultured separately for another $40 \mathrm{~h}$ in the presence or absence of TFP.

\section{Discussion}

Calmodulin, an ubiquitous and versatile calcium binding protein, is considered to be an important modulator of intracellular calcium functions (Cheung, 1980). Trifluoperazine selectively binds with calmodulin and inactivates various calmodulin-dependent actions of intracellular calcium actions (Connor et al., 1982; Prozialeck \& Weiss, 1982). If the effect of trifluoperazine on embryo development is considered to be mediated via alteration in calmodulin-dependent intracellular calcium functions, then we can postulate that extracellular and intracellular calcium-mediated events are essential for the process of compaction. Our results corroborate the findings of Bilozur $\&$ Powers (1982). However, while extracellular calcium appears to be necessary for the induction and continuous maintenance of compaction (Wales, 1970; Whitten, 1971; Ducibella \& Anderson, 1975; Bilozur \& Powers, 1982), the role of intracellular calcium seems to be associated mainly with induction and the initial phase of the compaction process. This belief is due to the fact that uncompacted embryos are unable to compact and that embryos, even at a late stage of compaction, can be induced to decompact in calcium-free medium, while trifluoperazine is only effective before and during the early compaction phase. Trifluoperazine had no effect upon advanced compacted embryos (Text-figs $2 \& 3$ ).

Whether calmodulin is present in the mouse preimplantation embryo or how calmodulincalcium-associated events could be involved in the compaction process and embryo development are not known. However, mouse oocytes contain high levels of calmodulin which is involved in oocyte maturation (Schultz, Heller \& Buklad, 1983). Calmodulin has been implicated in oocyte maturation in non-mammalian species (Meijer \& Guerrier, 1981; Wasserman \& Smith, 1981; Hollinger \& Alvarez, 1982). Of several possibilities, calmodulin-dependent adenylate cyclase does not appear to play any role in the compaction process (Bilozur \& Powers, 1982). However, calmodulin may modulate prostaglandin synthesis in the embryo through activation of phospholipase $\mathrm{A}_{2}$ (Wong \& Cheung, 1979) and prostaglandins thus generated may participate in embryo development (Biggers, Leonov, Baskar \& Fried, 1978). While a non-specific cytotoxic effect of trifluoperazine cannot be ignored completely, it seems unlikely because the drug was 
primarily effective in inhibiting the development of the uncompacted or the early compacted embryos. The birth of healthy young, following transfer of the blastocysts grown from the advanced compacted embryos in the presence of trifluoperazine to pseudopregnant mice, also suggests normal development of the embryos under these conditions. Furthermore, trifluoperazine sulphoxide, the inactive form of trifluoperazine, had no effect on the development of the 4-8-cell uncompacted embryos to blastocysts and on subsequent implantation after transfer to pseudopregnant mice.

The results suggest, therefore, that extracellular and intracellular calcium-related events participate in the induction of the compaction process. However, extracellular calcium is essential for continuous maintenance of the compaction process, while intracellular calcium is required only during the initial phase.

We thank Dr Michael Cohn, Department of Pharmacology, Duke University Medical Center, Durham, North Carolina, and Mr John Paul, Smith Kline \& French Laboratories, Philadelphia, Pennsylvania, U.S.A., for the generous gifts of trifluoperazine sulphoxide and trifluoperazine. This research was supported by an NICHD grant (HD-12304). P.L.P. is a Rockefeller Foundation postdoctoral fellow.

\section{References}

Atienza-Samols, S.B., Pine, P.R. \& Sherman, M.I. (1980) Effects of tunicamycin upon glycoprotein synthesis and development of early mouse embryos. Devl Biol. 79, 19-32.

Biggers, J.D., Leonov, B.V., Baskar, J.F. \& Fried, J. (1978) Inhibition of hatching of mouse blastocysts in vitro by prostaglandin antagonists. Biol. Reprod. 19, 519-533.

Bilozur, M. \& Powers, D. (1982) Two sites for calcium action in compaction of the mouse embryo. Expl Cell Res. 142, 39-45.

Cheung, W.Y. (1980) Calmodulin-an introduction. In Calcium and Cell Function, Vol. I, pp. 1-12. Ed. W. Y. Cheung. Academic Press, New York.

Connor, C.G., Levin, R. \& Brownstein, B.L. (1982) Effect of trifluoperazine on calcium uptake and calciumdependent functions in cultured epithelial cells. Biol. of the Cell 46, 37-42.

Dey, S.K. \& Johnson, D.C. (1980) Histamine formation by mouse preimplantation embryos. J. Reprod. Fert. 60, $457-460$.

Ducibella, T. \& Anderson, E. (1975) Cell shape and membrane change in the eight-cell mouse embryo: prerequisites for morphogenesis of the blastocyst. Devl Biol. 47, 45-48.

Hollinger, T.G. \& Alvarez, I.M. (1982) Trifluoperazine induced meiotic maturation in Xenopus laevis. J. exp. Zool. 224, 461-464.

Hudgins, L., Mukherjee, S. \& Dey, S.K. (1982) Preimplantation embryo development in the mouse : role of histidine decarboxylase. Gamete Res. 6, 121-125.

Meijer, L. \& Guerrier, P. (1981) Calmodulin in starfish oocytes. 1. Calmodulin antagonists inhibit meiosis reinitiation. Devl Biol. 88, 318-324.
Pratt, H.P.M., Keight, J. \& Chakraborty, J. (1980) Membrane sterols and the development of the preimplantation mouse embryo. J. Embryol. exp. Morph. 60, 303-319.

Prozialeck, W.C. \& Weiss, B. (1982) Inhibition of calmodulin by phenothiazines and related drugs: structure activity relationships. J. Pharmacol. exp. Ther. 222, 509-516.

Schultz, R.M., Heller, D.T. \& Buklad, N. (1983) Implication of a calmodulin-dependent step in mouse oocyte maturation. Biol. Reprod. 28, Suppl. 1, 138, Abstr. 212.

Surani, M.A.H. (1979) Glycoprotein synthesis and inhibition of glycosylation by tunicamycin in preimplantation mouse embryos: compaction and trophoblast adhesion. Cell 18, 217-227.

Wales, R.G. (1970) Effects of ions on the development of the preimplantation mouse embryo in vitro. Aust. $J$. biol. Sci. 23, 421-429.

Wasserman, W.J. \& Smith, L.D. (1981) Calmodulin triggers the resumption of meiosis in amphibian oocytes. J. Cell Biol. 89, 389-394.

Weiss, B. \& Wallace, T.L. (1980) Mechanisms and pharmacological implications of altering calmodulin activity. In Calcium and Cell Function, Vol. I, pp. 329379. Ed. W. Y. Cheung. Academic Press, New York.

Whitten, W.K. (1971) Nutrient requirements for the culture of preimplantation embryos in vitro. Adv. Biol. Sci. 6, 129-141.

Wong, P.Y.K. \& Cheung, W.Y. (1979) Calmodulin stimulates human phospholipase $A_{2}$. Biochem. Biophys. Res. Commun. 90, 473-480.

Received 27 October 1983 